\title{
Ensayo a escala de obra sobre fisuración y agrietamiento
}

\author{
J. I. Martinez ynzenga y M. Aguanell \\ Portland Valderribas, s. A.
}

Con objeto de demostrar que el hormigón confeccionado con cemento PA-350 no presenta ninguna anomalía (si se pone en obra y se siguen las reglas elementales de cualquier código de buena práctica y que especifican todas las Normas referentes al hormigón, las cuales se dan para cumplir en todos los hormigones, independientemente del cemento empleado en la confección del mismo), se pensó realizar un ensayo a escala de obra, sobre una losa de pavimento, para reproducir unas condiciones donde la relación superficie a volumen fuera grande.

De nuestra propia experiencia y de toda la bibliografía leída sabíamos que el principal agente productor de las grandes grietas que se forman cuando todavía está el hormigón en estado plástico, y que empiezan a aparecer aproximadamente a la hora u hora y media, después de colocado el hormigón, es la velocidad de evaporación. Con estos conocimientos $\mathrm{y}$, siendo las corrientes de aire uno de los elementos que mayor evaporación pueden producir (dependiendo, naturalmente, de la humedad relativa del mismo), decidimos que una mitad de la losa que proyectábamos realizar fuera sometida a una corriente de aire, producida por dos ventiladores de $1 / 2 \mathrm{CV}, 1.400$ r.p.m., y la otra mitad estuviera al abrigo del aire, pero que no se le daría ningún tratamiento especial, ni siquiera un segundo fratasado, ni ningún riesgo hasta después de pasadas, por lo menos, 24 horas.

Las dimensiones de la losa fueron de 6,90 × 4,20 × 0,26 m, y se dispuso un tabique de lámina de plástico dividiéndoila en dos mitades aproximadamente, según ilustra la figura 1. A la losa, en vez de apoyarla directamente sobre el terreno, se le intercaló una lámina de plástico, con objeto de evitar la absorción que pudiera hacer el terreno soportante.

El hormigón que se utilizó para confeccionar la losa fue suministrado por una Central Hormigonera, con una dosificación de $370 \mathrm{~kg}$ de cemento PA-350 por metro cúbico; los áridos estaban en las proporciones de $60 \%$ de grava, canto rodado, tamaño máximo 20 $\mathrm{mm}$ y $40 \%$ de arena, con un módulo de finura de 3,10, el asentamiento en el cono de Abrams fue de $8 \mathrm{~cm}$.

Este hormigón estaba diseñado para poder ser bombeado.

Se empezó a poner el hormigón en obra a las 12 horas, se vibró con vibrador de aguja de $8.000 \mathrm{~Hz}$. Se llenó el encofrado con dos cubas, una a continuación de la otra, empleando en esta operación 20 minutos. Inmediatamente después se enrasó con una regla maestra, imprimiéndole un movimiento de sierra. Terminado este proceso se montó la pantalla o tabique de plástico, se pusieron los ventiladores en marcha y ya no se volvió a tocar la superficie de la losa. 
La humedad relativa del aire fue del $43 \%$; temperatura ambiente, $16,5^{\circ} \mathrm{C}$ (temperatura termómetro seco); la temperatura del termómetro húmedo del psicómetro, $10,5^{\circ} \mathrm{C}$.

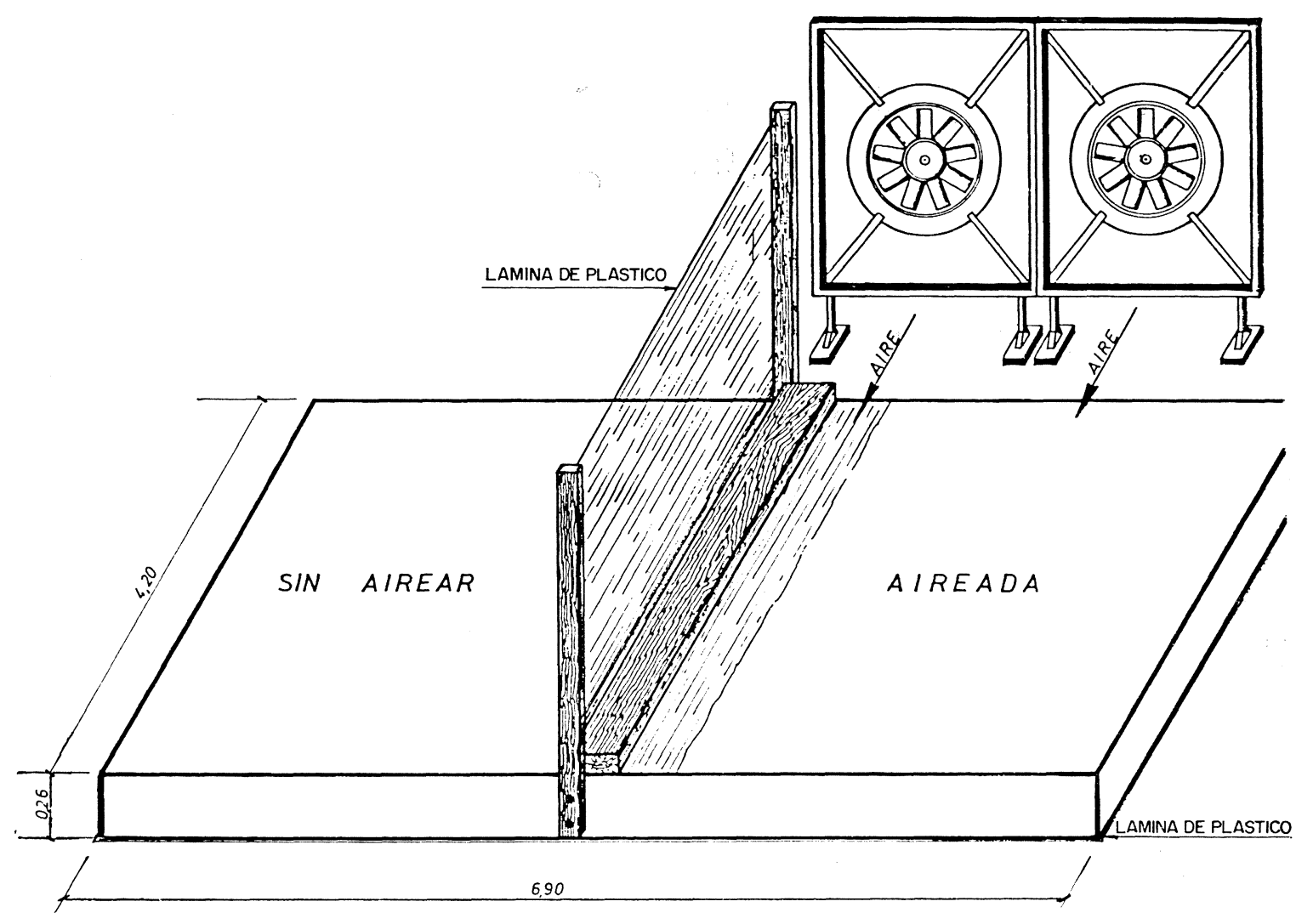

Fig. 1

A las $2^{1 / 2}$ de la tarde, o sea 1 hora 30 minutos después de que fue enrasada la superficie del hormigón, empezaron a manifestarse en la parte sometida a la aireación, únicamente, formaciones de grietas; a las 2 horas eran más acusadas y, a las 3 horas, aún más, quedándose estabilizadas en este tiempo.

La mitad que estuvo al abrigo del aire no presentó ningún síntoma de agrietamiento, a pesar, como hemos dicho en un principio, de que no se le aplicó ningún tratamiento (fig. 2).

Es de todos conocido, que las fisuras que se originan en el hormigón, debidas a la retracción de secardo, son de dos tipos:

1. Las que se producen en estado plástico del hormigón y antes de finalizar el fraguado (retracción plástica).

2. Las que se producen en el hormigón endurecido (retracción hidráulica).

Sólo las primeras, de las cuales trataremos en este trabajo, son de mucha mayor importancia, ya que más que fisuras son grietas, de mucha anchura y mucha profundidad.

En los casos vistos y estudiados por nosotros, siempre nos hemos encontrado que la profundidad de la grieta afectaba a todo el espesor de la losa o del forjado, y que las anchuras de la misma, en la parte superior, pueden llegar a tener hasta $3 \mathrm{~mm}$. Estas grietas son las que llama el Sr. Joisel en su libro "Fisuras y Grietas en Morteros y Hormigones", pág. 41 (de la cuarta edición), grietas por Acomodación, y a la que los sajones llaman Retracción Plástica. 
Para comprobar la profundidad de la grieta se sacaron testigos. Las dos fotografías de las figuras 3 y 4 son de los huecos que dejaron las extracciones de los testigos; en ellas se puede apreciar cómo la grieta va desde la superficie hasta el fondo.

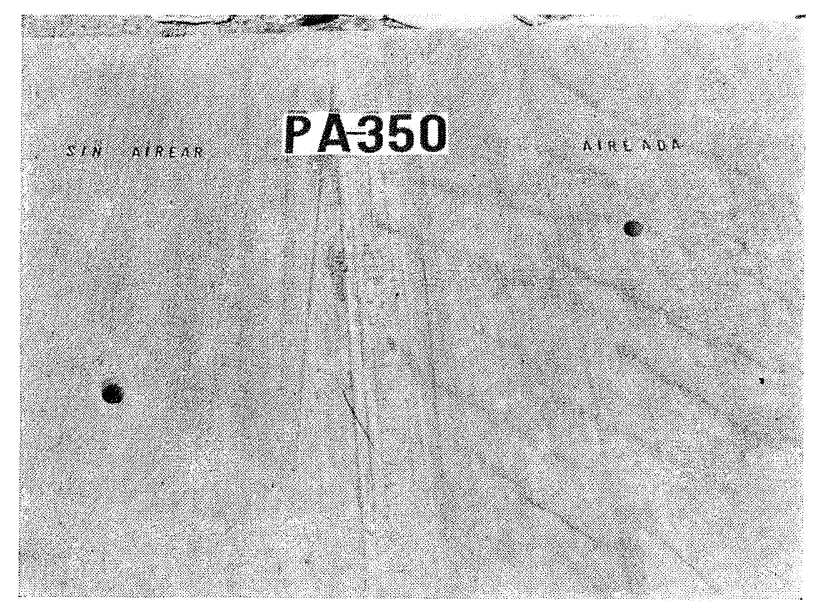

Fig. 2

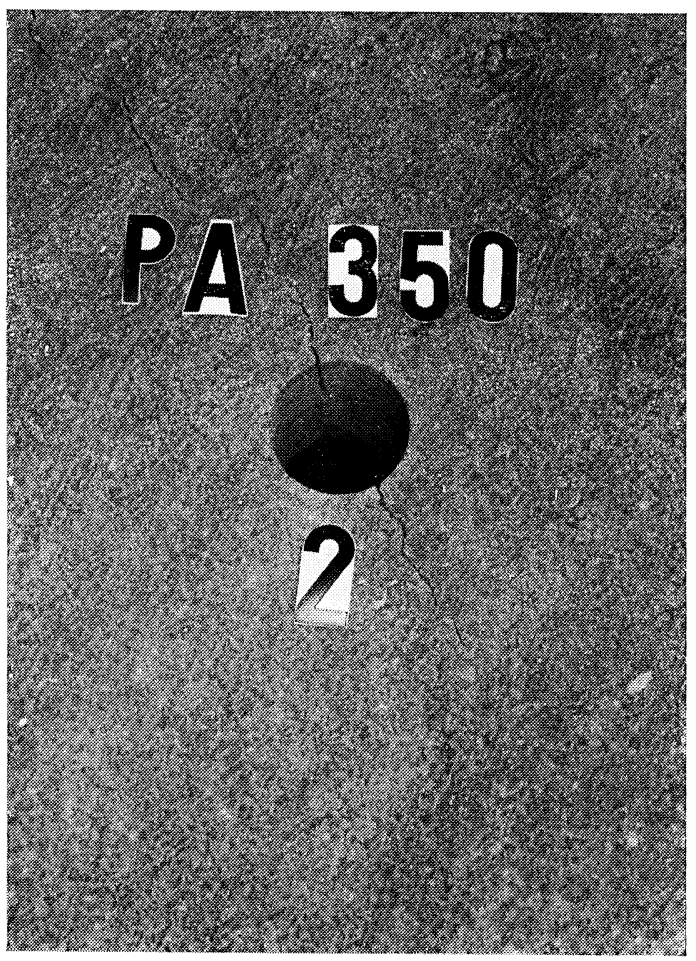

Fig. 4

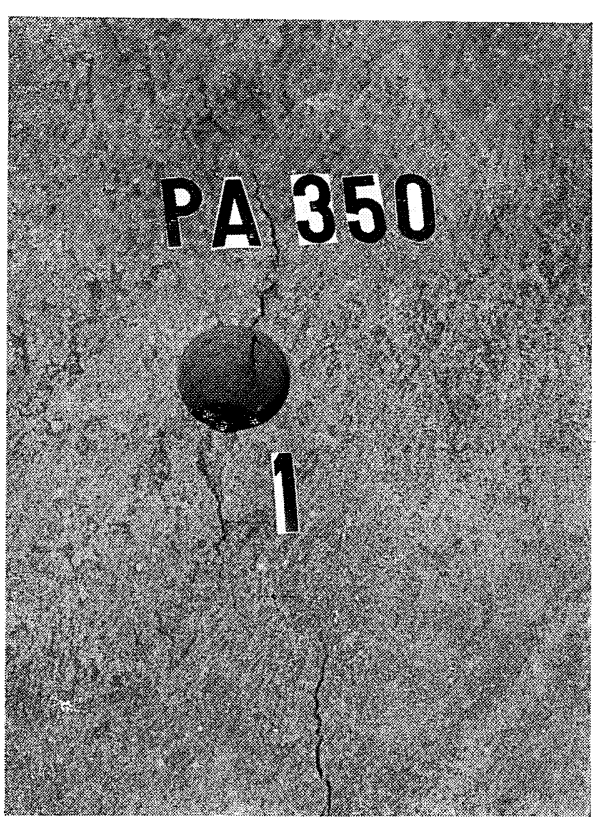

Fig. 3

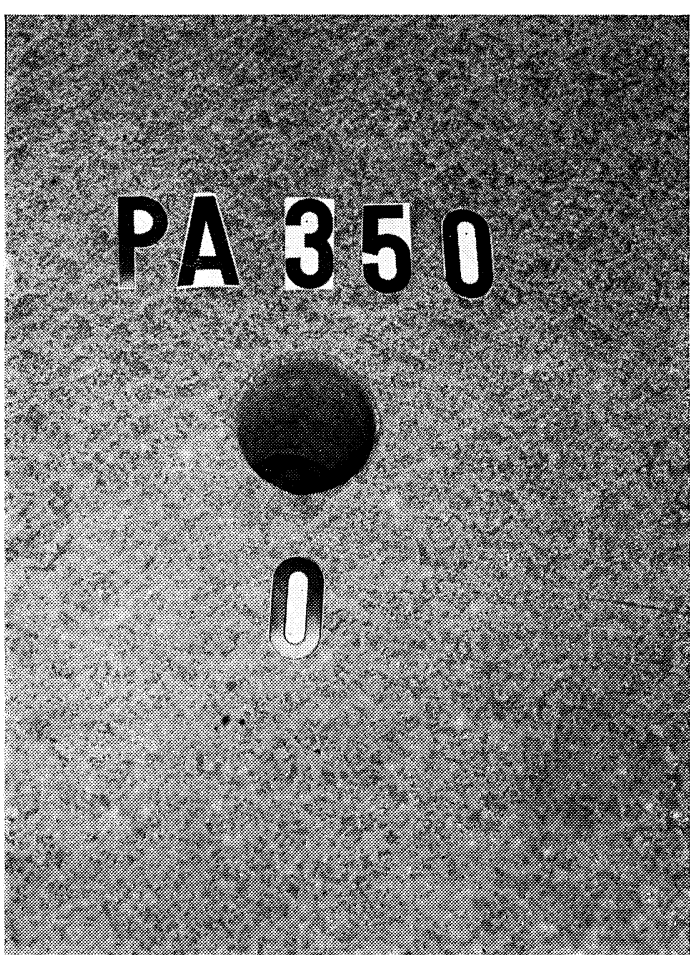

Fig. 5 
En la fotografía de la figura 5 corresponde a un testigo extraído de la parte de losa que no fue sometida a la corriente de aire. No se aprecia ningún síntoma, ni de grieta ni fisuración, tanto superficial como interna.

Posteriormente, se volvió a repetir el ensayo con un hormigón de las mismas características que el anterior, pero sustituyendo el cemento PA-350 por cemento P-350. La misma dosificación, las mismas proporciones de arena y grava y el mismo asiento.

La colocación en obra se hizó también por la mañana. En este caso, la humedad relativa del aire fue del $65 \%, 17^{\circ} \mathrm{C}$ temperatura-termómetro seco (temperatura ambiente) y $13,5^{\circ} \mathrm{C}$ temperatura del termómetro húmedo.

En las fotografías de las figuras 6 a 9 se puede observar que el fenómeno se repite; y si las grietas no son tan numerosas como en el caso precedente del PA-350 es debido a que el aire que pasó por la superficie de la losa estaba más saturado de humedad y su poder de desecación fue menor en este segundo ensayo. No obstante, las grietas fueron tan profundas como en el primer caso.



Fig. 6

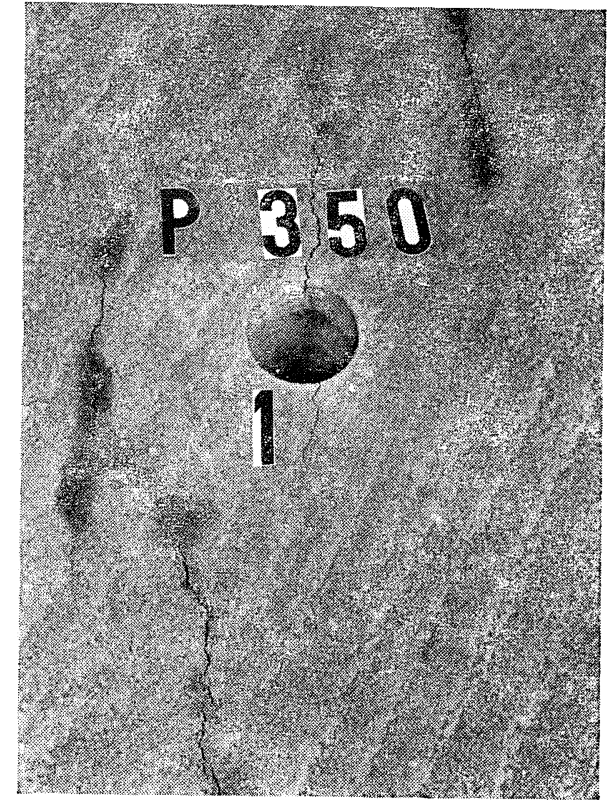

Fig. 7

La formación de estas grietas depende en buena medida de la velocidad de evaporación del agua de exudación del hormigón, independientemente de la naturaleza del conglomerante.

Se llega así a la conclusión de que, si se cumplen las normas del código de buena práctica del hormigón evitando una desecación rápida, por los muchos métodos que hay en uso, no aparece la formación de grietas, ya que en nuestro ensayo, con sólo evitar las corrientes de aire, que barren la superficie del hormigón, ha sido suficiente para que no 
aparezca grieta alguna; y si, además se hubiera cubierto con arpilleras mojadas, o regado con agua pulverizada o tratado con pinturas de curado, o inundado con una capa fina de agua, las garantías de eliminar las grietas sería total.

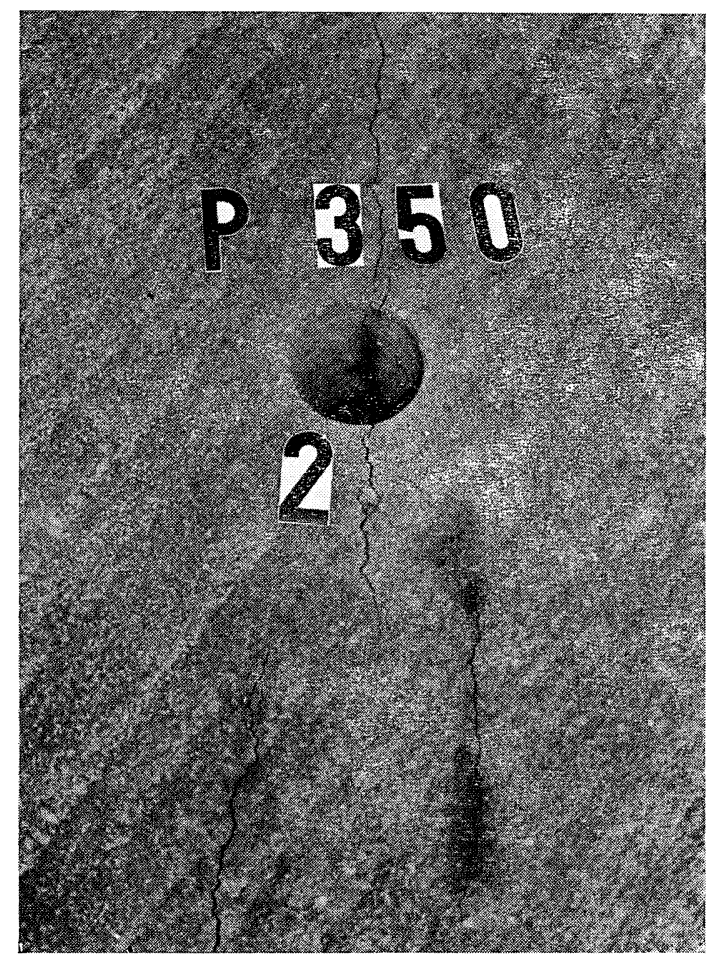

Fig. 8

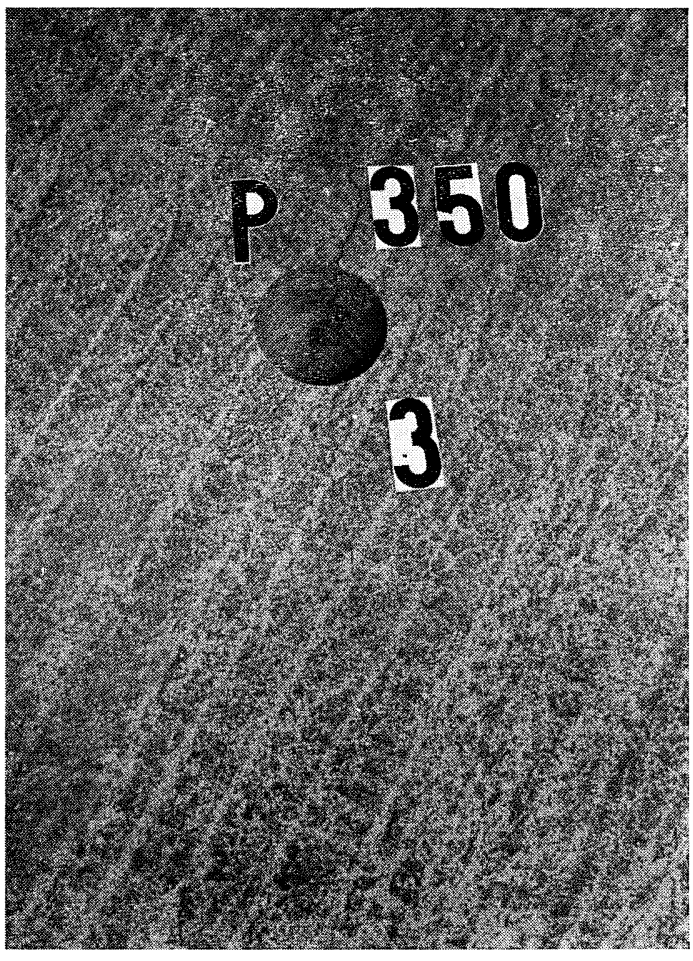

Fig. 9 